Tema: Solidificação / Lingotamento

\title{
CARACTERIZAÇÃO DE PÓS FLUXANTES UTILIZANDO TERMODINÂMICA COMPUTACIONAL, DIFRAÇÃO DE RAIOS-X E ANÁLISE TÉRMICA DIFERENCIAL*
}

\author{
Letícia Miranda Alves ${ }^{1}$ \\ Cynara Christ Klippe ${ }^{1}$ \\ Sábata Marla Reis Durães Oliveira ${ }^{2}$ \\ Altemar Dettogne do Nascimento ${ }^{3}$ \\ José Roberto de Oliveira ${ }^{4}$ \\ Estéfano Aparecido Vieira ${ }^{4}$
}

\section{Resumo}

Os fluxantes são misturas de minerais que em contato com aço irão fundir e gerar uma escória líquida que irá permitir a troca térmica e favorecer a lubrificação ao longo da interface placa/molde. Durante o processo de lingotamento contínuo de placas parte do filme de escória irá solidificar-se tendendo a formar fases amorfas e/ou cristalinas. O aumento na temperatura de solidificação bem como a formação de camada cristalina reduz a transferência de calor e pode repercutir em uma pele de aço mais fina favorecendo o breakout. Por outro lado, estas condições, como promovem uma solidificação do aço mais cuidadosa reduz a formação de trincas longitudinais a quente, depressões entre outros defeitos. Neste trabalho usando o software Thermocalc ${ }^{\circledR}$ simulou-se a formação de fases para dois tipos de pós fluxantes em função da temperatura. Usando o software FactSage estimaram-se os valores de viscosidade. Através de análise por difração de raios- $x$, microscopia eletrônica de varredura e análise térmica diferencial, buscou-se estabelecer as relações entre as propriedades e resultados esperados para cada tipo de pó fluxante no processo de lingotamento contínuo de placas.

Palavras-chave: Lingotamento contínuo; Aços; Peritéticos; Pó fluxante.

\section{FLUX POWDER CHARACTERIZATION BY USE OF COMPUTATIONAL THERMODYNAMIC, X-RAY DIFFRACTION AND THERMAL ANALYSIS}

\section{Abstract}

The mold powders are mixtures of minerals that in contact with molten steel will melts and produce a liquid slag which will allow the heat transfer and promote lubrication along the slab/mold interface. In this work using Thermocalc $₫$ software it was simulated the formation of phases for two types of mold powders as function of temperature. Using the software FactSage ${ }^{\circledR}$ were evaluated viscosity values. Through x-ray diffraction analysis, scanning electron microscopy and thermal analysis it was possible determine relationships between the properties and expected results for each type of mold flux powder used in slab continuous casting process.

Keywords: Continuous casting; Hot cracks; Peritectic; Mold powder.

Engenheiro Metalurgista, IFES, Campus Vitória, ES, Brasil.

Mestranda em Eng. Metalúrgica e de Materiais, PROPEMM, IFES, Campus Vitória, ES, Brasil.

M.Sc. Engenharia, Especialista de Lingotamento Contínuo, ArcelorMittal Tubarão, Serra, ES, Brasil.

4 Doutor em Engenharia Metalúrgica e de Materiais, PROPEMM, IFES, Campus Vitória, ES, Brasil.

* Contribuição técnica ao 45 Seminário de Aciaria - Internacional, 25 a 28 de maio de 2014, Porto Alegre, RS, Brasil. 


\section{INTRODUÇÃO}

No desenvolvimento de pós fluxantes para o lingotamento contínuo de aços é indispensável buscar condições de solidificação que permitam maximizar a velocidade dos veios associado a uma boa qualidade dos produtos. As condições de solidificação no molde têm importância fundamental e os pós fluxantes influenciam principalmente nos seguintes aspectos: i) lubrificação entre o molde e casca solidificada; ii) transferência de calor entre o molde e placa; iii) isolação térmica no topo do molde; iv) proteção contra a oxidação; v) absorção de inclusões na superfície. Estes parâmetros terão um efeito decisivo na qualidade e na produtividade [1].

Durante a operação de lingotamento de placas, o pó fluxante é continuamente adicionado no topo do molde sobre a poça de aço fundido e forma-se uma camada líquida com uma profundidade aproximada de 10 a $25 \mathrm{~mm}$. Normalmente o valor mínimo recomendado é 1,5 vezes a amplitude do oscilador do molde. Devido à temperatura elevada do aço que é superior a temperatura de fusão do fluxante, temse então um gradiente na direção vertical no fluxante fundido. No topo o pó fica seco e livre de materiais voláteis. Logo abaixo forma-se uma camada de partículas sinterizadas e este sistema garante uma boa isolação térmica e por fim tem-se uma camada do fluxo fundido. O líquido formado penetra entre o gap deixada pelo molde e a placa que está se solidificando. Uma fina camada vítrea é formada quando o fluxo entra em contato com a parede do molde que é resfriado com água. Dependendo das condições de resfriamento e características da composição química do fluxante, existe a possibilidade de ocorrer a formação de fases cristalinas no filme solidificado [2]. A formação do filme sólido com fase cristalina tem forte influência na transferência de calor que por sua vez tem efeito direto na quantidade e nos tipos de defeitos que podem se formar [3].

Dependendo das condições de solidificação as trocas térmicas no molde podem ser não uniformes. Os gradientes térmicos podem surgir devido a cristalização parcial do pó fluxante [4] e a cuspidina $\left(3 \mathrm{CaO} .2 \mathrm{SiO}_{2} \cdot \mathrm{CaF}_{2}\right)$ é um exemplo de composto ternário que pode cristalizar-se em vários sistemas [1,5-7]. O esfriamento não uniforme pode favorecer a formação de trincas entre outros defeitos devido ao acumulo de tensões. O controle de formação de algumas fases específicas para os pós-fluxantes pode ser feito através do uso de diagramas para transformações isotérmicas (TTT) $[8,9]$ e também através de diagramas sob resfriamento continuo (CCT) [10] no entanto, poucos trabalhos com medições específicas estão disponíveis na literatura [1]. $\mathrm{Na}$ falta destes dados, uma maneira de tentar prever o comportamento de um fluxante durante o lingotamento contínuo é através da caracterização do mesmo e assim, o objetivo deste trabalho foi caracterizar dois pós fluxantes comerciais usando a termodinâmica computacional, análise térmica diferencial (DSC), difração de raios- $x$ e microscopia eletrônica de varredura. As informações obtidas permitem especificar com maior segurança a aplicação dos fluxantes bem como estabelecer as condições limites de processo.

\section{MATERIAIS E MÉTODOS}

Para o presente estudo dois tipos de fluxantes foram testados: Fluxante $A$ com basicidade binária $B=1,41$ e Fluxante $B$ com $B=0,93$. $A$ composição química média foi inserida no Software Thermocalc $®$ para simular as prováveis fases que se formam durante o aquecimento. A base de dados foi o Slag3. Através do uso do

\footnotetext{
* Contribuição técnica ao $45^{\circ}$ Seminário de Aciaria - Internacional, 25 a 28 de maio de 2014, Porto Alegre, RS, Brasil.
} 
Software FactSage estimou-se a viscosidade em função da temperatura. A Tabela 1 apresenta a composição química média fornecida pelo fabricante.

Tabela 1. Composição química média dos pós fluxantes (\% em peso)

\begin{tabular}{|ccccccc|}
\hline & $\mathrm{SiO}_{2}$ & $\mathbf{C a O}$ & $\mathbf{A l}_{2} \mathbf{O}_{3}$ & $\mathbf{N a}_{2} \mathbf{O}$ & $\mathbf{F}$ & $\mathbf{C}_{\text {total }}$ \\
\hline Fluxante A & 30 & 43 & 4 & 4 & 10 & 5 \\
Fluxante B & 33 & 31 & 3 & 10 & 8 & 5 \\
\hline
\end{tabular}

\subsection{Microscopia Eletrônica de Varredura e EDS}

Os pós não aglomerados e aglomerados foram submetidos à análise microestrutural por microscopia eletrônica de varredura (MEV) utilizando-se um equipamento da Marca Zeiss Mod. Evo 10. Microanálises químicas foram realizadas usando EDS (Energy Dispersive Spectroscopy) da Marca Oxford.

\subsection{Difração de Raios-X}

Para conhecer quais são as fases presentes os pós desaglomerados e peneirados foram submetidos à análise por difração de raios-X. Foi utilizado um equipamento da marca Bruker Mod. D2Phaser com goniômetro tipo Bragg-Brentano com angulo $2 \theta$ variando de 10 até $80^{\circ}$, passo de $0,02^{\circ}$ com tempo de leitura de $2 \mathrm{~s}$ e rotação de $10 \mathrm{rpm}$.

\subsection{Análise Térmica Diferencial (TG-DSC)}

Para determinar as temperaturas onde ocorrem as transformações alotrópicas, as amostras foram submetidas à análise térmica diferencial (Differential Scanning Analysis-TG-DSC) em um equipamento da marca Netzsch Mod. STA 449 F3 Jupiter ${ }^{\circledR}$. Os pós foram desaglomerados usando um recipiente de porcelana e em seguida peneirado até que todo material atravessasse uma peneira com malha inferior a 200 mesh. Em seguida, cerca de 40mg de cada tipo de fluxante foram pesados em uma balança de precisão. As amostras foram acondicionadas em um cadinho de platina e levadas ao aquecimento no TG-DSC até uma temperatura de $1400^{\circ} \mathrm{C}$ sob uma taxa de $10^{\circ} \mathrm{C} / \mathrm{min}$. Em seguida, procedeu-se o resfriamento na mesma velocidade. Para proteger as amostras durante as medições, utilizou-se argônio analítico 5.0.

\section{RESULTADOS E DISCUSSÕES}

A Figura 1 apresenta os resultados obtidos usando o software Thermocalc ${ }^{\circledR}$ e são apresentadas as prováveis fases que se formam durante o aquecimento. Ambos fluxantes fundem-se por completo por volta de $1300^{\circ} \mathrm{C}$ porém, diferente do fluxante $A$, os resultados prevêem para o fluxante $B$ a formação de duas escórias imiscíveis a partir de aproximadamente $1100^{\circ} \mathrm{C}$. A $1400^{\circ} \mathrm{C}$ os líquidos formados possuem a seguinte composição: Fluxo B1: 40,0\% CaO, 24,4\% $\mathrm{CaF}_{2}, 2,8 \% \mathrm{Na}_{2} \mathrm{O}, 22,8 \% \mathrm{SiO}_{2}$, $4,6 \% \mathrm{Al}_{2} \mathrm{O}_{3}$. Fluxo B2: $21,2 \% \mathrm{CaO}, 1,7 \% \mathrm{CaF}_{2}, 25,0 \% \mathrm{Na}_{2} \mathrm{O}, 51,5 \% \mathrm{SiO}_{2}, 0,6 \%$ $\mathrm{Al}_{2} \mathrm{O}_{3}$. A formação de líquidos imiscíveis já foi observada para outras composições por Rosa e Lanyi em 1981 [11], mas é difícil estabelecer que tipo de comportamento os fluídos formados terão no molde com relação as condições de lubrificação, fluxo e troca de calor. Um típico molde para placas possui aproximadamente $900 \mathrm{~mm}$ de

\footnotetext{
* Contribuição técnica ao 450 Seminário de Aciaria - Internacional, 25 a 28 de maio de 2014, Porto Alegre, RS, Brasil.
} 
altura e nestas condições na saída, uma típica temperatura na superfície simulada por Meng e Thomas em 2003 [12] é de aproximadamente $1100^{\circ} \mathrm{C}$. Nesta situação, a fração de líquido presente usando o fluxante $A$ é de 0,40 contra apenas 0,25 do fluxante B. Portanto, sob este aspecto, existe a tendência do sistema de lubrificação para o fluxante $A$ ser mais eficiente já que a fração de líquido é maior, além disso, o fluxante A apresenta viscosidade inferior ao fluxo B1 (SLAG1 - Figura 1) como será mostrado posteriormente. Para temperaturas superiores a $1120^{\circ} \mathrm{C}$, a situação se inverte e o fluxante A passa ter uma fração em torno de 0,41 contra 0,54 para o fluxante $B$. A presença de fase sólida no fluxo tem um impacto significativo no valor final da viscosidade e tende a aumentá-la. No entanto percebemos que o fluxante $B$ forma as duas escórias distintas e imiscíveis e pouco se sabe como é o comportamento de dois líquidos imiscíveis sob estas condições. Uma análise mais cuidadosa deveria ser feita para prever também a formação de fases cristalinas já que os diagramas apresentados na Figura 1 são sob condições de equilíbrio e, portanto, não representam as situações reais para prever também como será a troca de calor mas, é consenso que fluxantes com maior quantidade de $\mathrm{CaO}$ e maior presença de $\mathrm{CaF}_{2}$ possuem maior tendência a formar fases cristalinas e portanto tem-se um sistema que terá uma troca térmica mais amena, sendo assim, para uma mesma velocidade de lingotamento a tendência são maiores temperaturas na saída do molde favorecendo melhores condições de lubrificação mas aumenta-se o risco de rompimento de pele. Somente do ponto de vista da viscosidade é provável que o fluxante A trará melhores condições de deslizamento principalmente nas situações onde a temperatura no final do molde seja inferior a $1100^{\circ} \mathrm{C}$ pois, a fração sólida presente será menor.
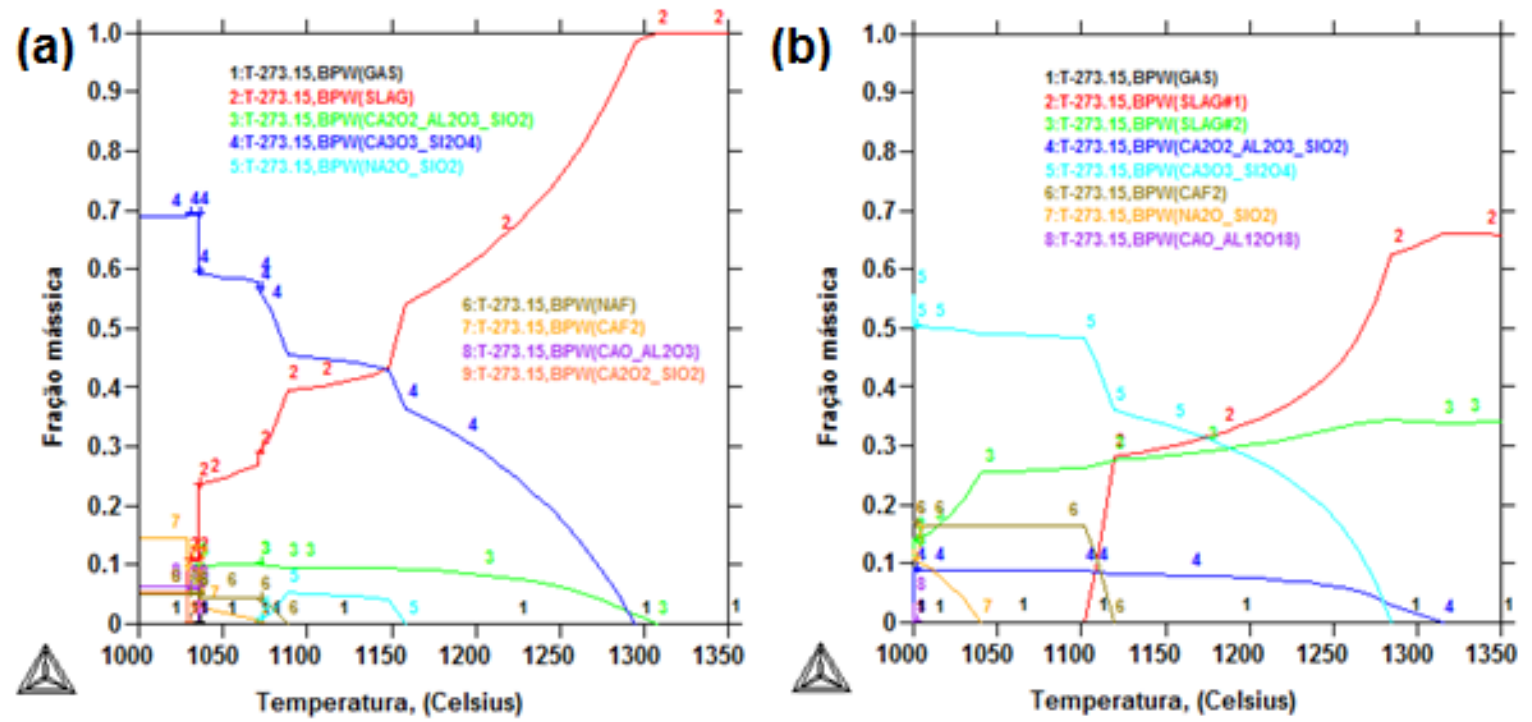

Figura 1. Evolução da formação de fases dos fluxantes em função da temperatura simulado no software Thermocalc ${ }^{\circledR}$, base slag3. (a) fluxante $A$ com basicidade binária $B=1,41$ e $F$ igual a 9,5\%. (b) fluxante $B$ com basicidade binária $B=0,93$ e $F$ igual a $7,5 \%$.

A Figura 2 correlaciona a viscosidade em função da temperatura para cada tipo de fluxante. O software FactSage ${ }^{\circledR}$ realiza uma estimativa seguindo a bem estabelecida equação de Arrhenius e as energias de ativação obtidas variaram de 47,9 até 42,8 $\mathrm{kcal} / \mathrm{K}$.mol. A simulação computacional indica que o Fluxante A é um pouco mais sensível a temperatura. Conforme já foi explicado, no caso do fluxante B com a presença de dois líquidos imiscíveis é difícil determinar qual será o comportamento

\footnotetext{
* Contribuição técnica ao 450 Seminário de Aciaria - Internacional, 25 a 28 de maio de 2014, Porto Alegre, RS, Brasil.
} 
reológico da mistura, em especial sob uma situação onde temos a constante troca de calor podendo ou não haver a formação de fases complexas. Para ligas metálicas binárias onde os elementos formam somente um líquido, a viscosidade de uma mistura pode ser estimada facilmente pela relação linear:

$$
\eta_{\text {mix }}=X_{A} \cdot \eta_{A}+X_{B} \cdot \eta_{B}
$$

Onde $\eta_{\text {mix }}$ é a viscosidade da liga, $\eta_{A}$ é a viscosidade do metal $A$ e $\eta_{B}$ é a viscosidade do metal $B, X_{A}$ e $X_{B}$ representam as frações molares de cada metal. Este modelo foi sugerido por Thresh and Crawleyl [13] em 1970 para ligas de Pb-Sn. Contudo, não funciona para qualquer liga metálica e Ganesan et al. [14] em 1987 sugeriu uma outra função matemática empírica para a liga binária $\mathrm{Al}-\mathrm{Cu}$. O fato é que conforme relata Seetharaman e Sichen [15], a viscosidade dos líquidos são funções de suas ligações, estruturas e configurações de suas espécies. Uma vez que estes parâmetros estão diretamente correlacionados com propriedades termodinâmicas e termofísicas é esperado que a viscosidade se relacione também com eles.
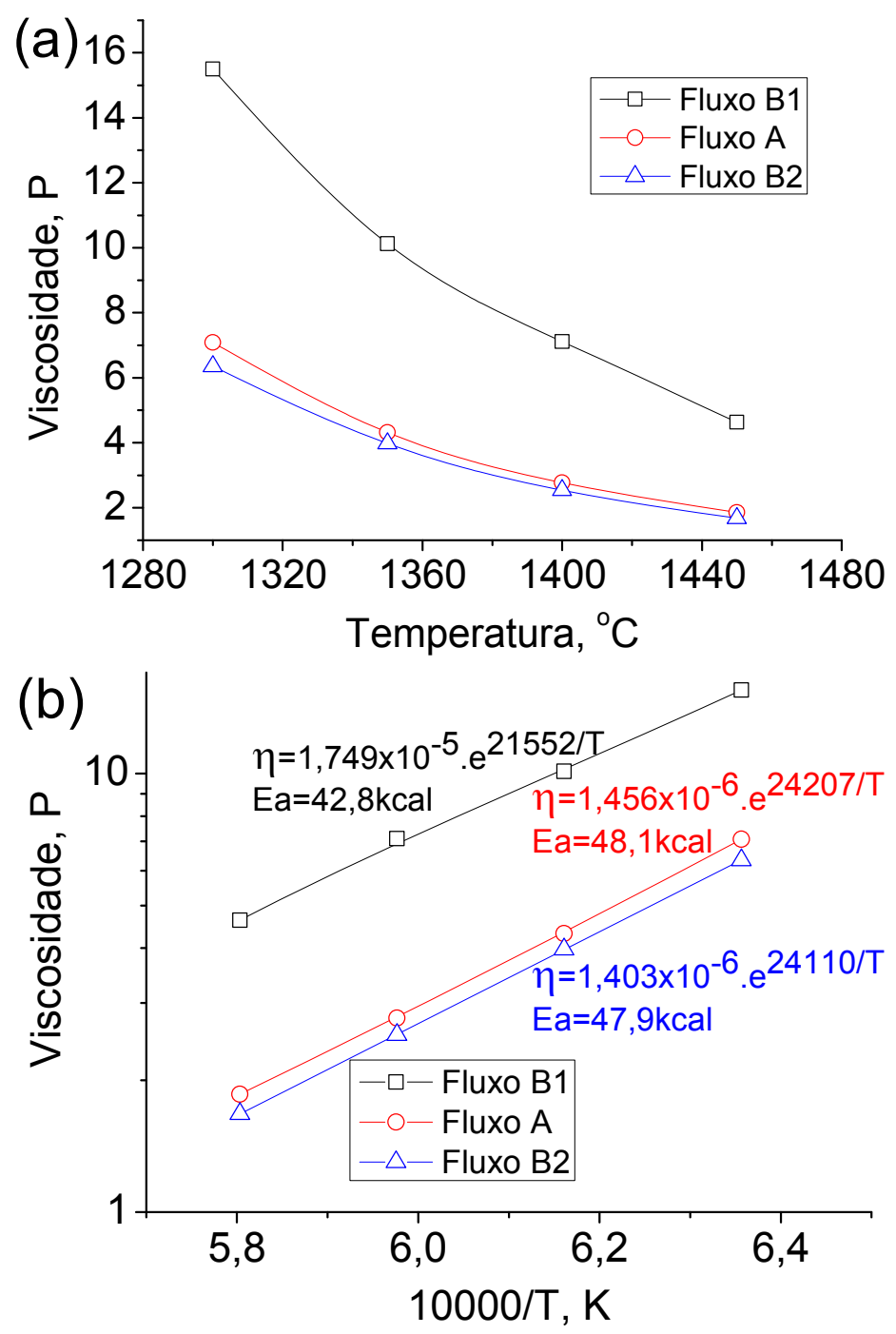

Figura 2. Evolução da viscosidade em função da temperatura para os fluxantes.

Existem muitos trabalhos e modelos que visam prever a viscosidade de misturas como o proposto por Moelwyn-Hugues [16] em 1961 para sistemas binários, Seetharaman e Sichen [13] para sistemas iônicos fundidos e outros mais modernos

\footnotetext{
* Contribuição técnica ao 450 Seminário de Aciaria - Internacional, 25 a 28 de maio de 2014 Porto Alegre, RS, Brasil.
} 
[17]. Entretanto, estes modelos não se mostram satisfatórios para todos os sistemas. Os trabalhos que buscam prever a viscosidade para misturas iônicos/covalentes fundidas a partir de propriedades fundamentais são restritos e complexos e por isso, existe um número significativo de modelos semi-empíricos os quais aplicam-se a condições bastante específicas. Por isso, nos tempos atuais as viscosidades de misturas precisam ser estudadas de forma independente caso a caso.

A Figura 3 apresenta o aspecto dos pós fluxantes como fabricados e percebem-se aglomerados de morfologia esférica com aproximadamente $0,5 \mathrm{~mm}$ de diâmetro que são compostos por partículas menores com dimensões inferiores a $100 \mu \mathrm{m}$. Do ponto de vista qualitativo as micro-análises estão de acordo com a especificação do fabricante e foi possível confirmar que o fluxante $\mathrm{A}$ possui maior quantidade de $\mathrm{F} e$ também maior basicidade binária logo é o mais indicado para ligas peritéticas. As Figuras 4 e 5 reforçam este resultado através da apresentação do mapeamento das partículas presentes por composição química e os picos referentes aos níveis energéticos dos elementos encontrados respectivamente. $O$ excesso de carbono mostrado na Figura 5 pode ser explicado pelo uso do embutimento de resina. As medidas de análise química por EDS são pouco confiáveis, em especial com relação a elementos leves, entretanto, atualmente os equipamentos modernos estão sendo aperfeiçoados.
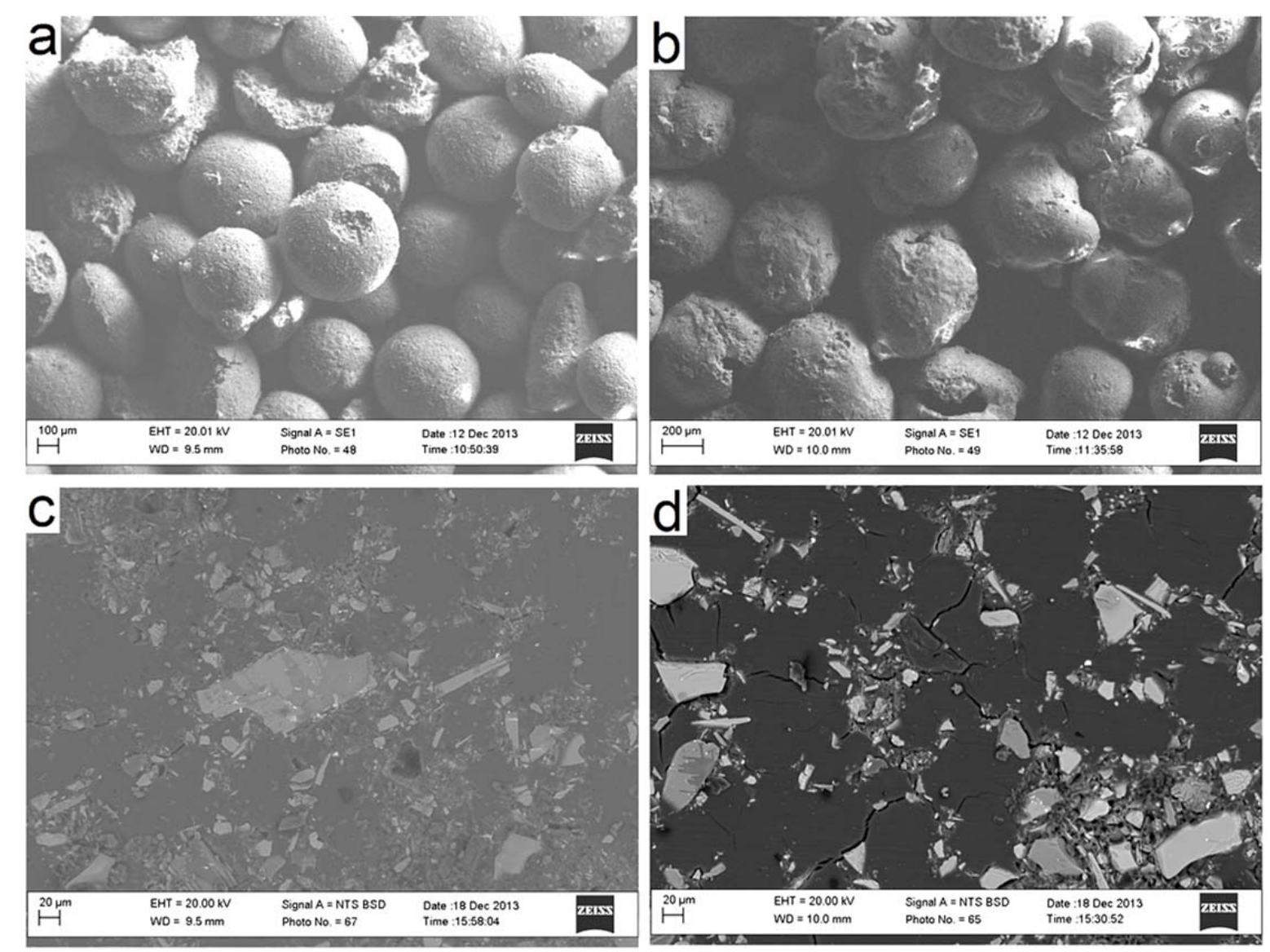

Figura 3. Típicas microestruturas para os pós fluxantes. (a) e (b) na sua forma aglomerada pronto para uso no processo. Imagens (c) e (d) mostram as micro partículas que formam os aglomerados. Imagens (a) e (c) fluxante A e (b) e (d) fluxante B.

\footnotetext{
* Contribuição técnica ao 45 Seminário de Aciaria - Internacional, 25 a 28 de maio de 2014, Porto Alegre, RS, Brasil.
} 

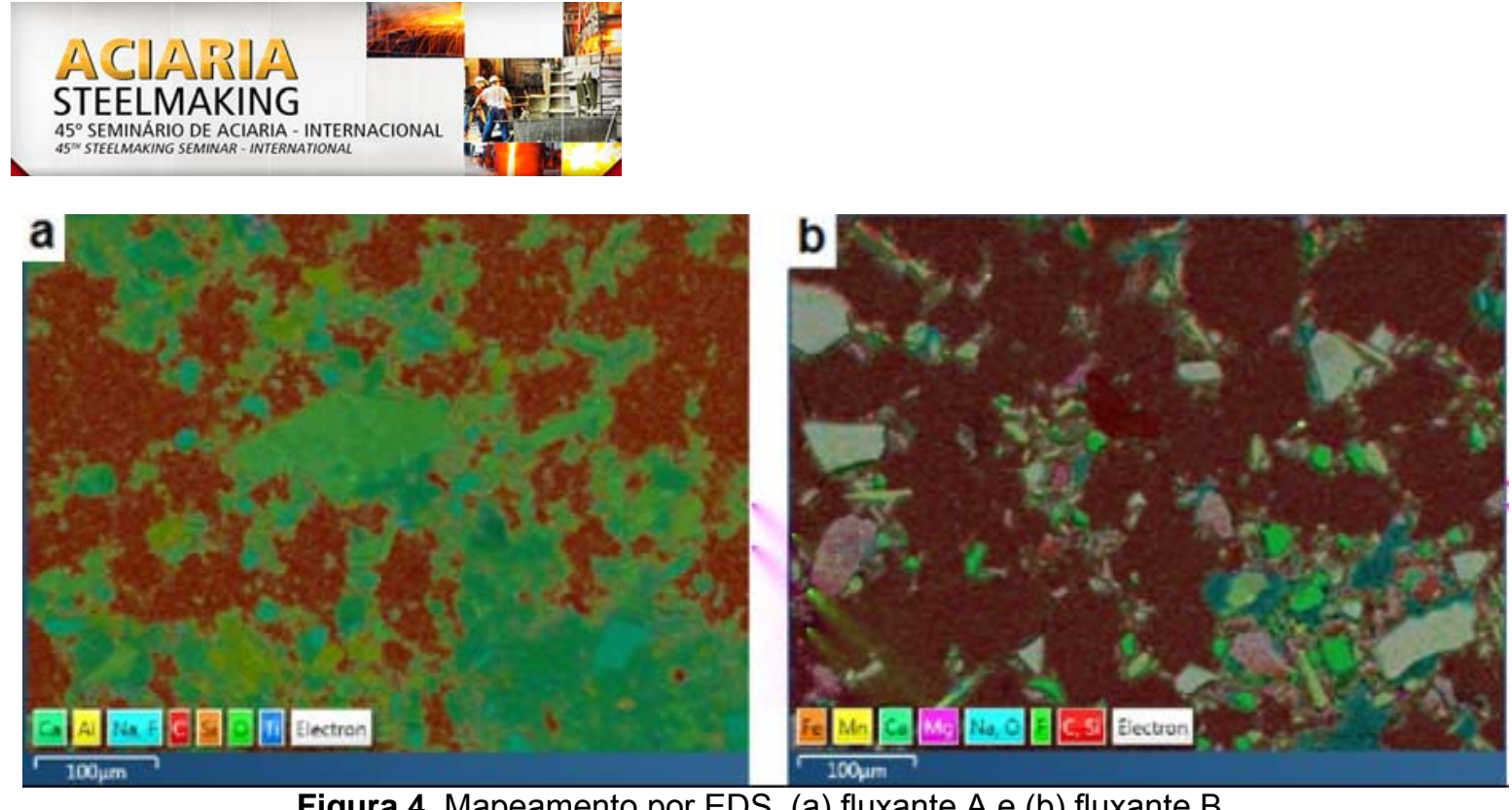

Figura 4. Mapeamento por EDS. (a) fluxante A e (b) fluxante B.
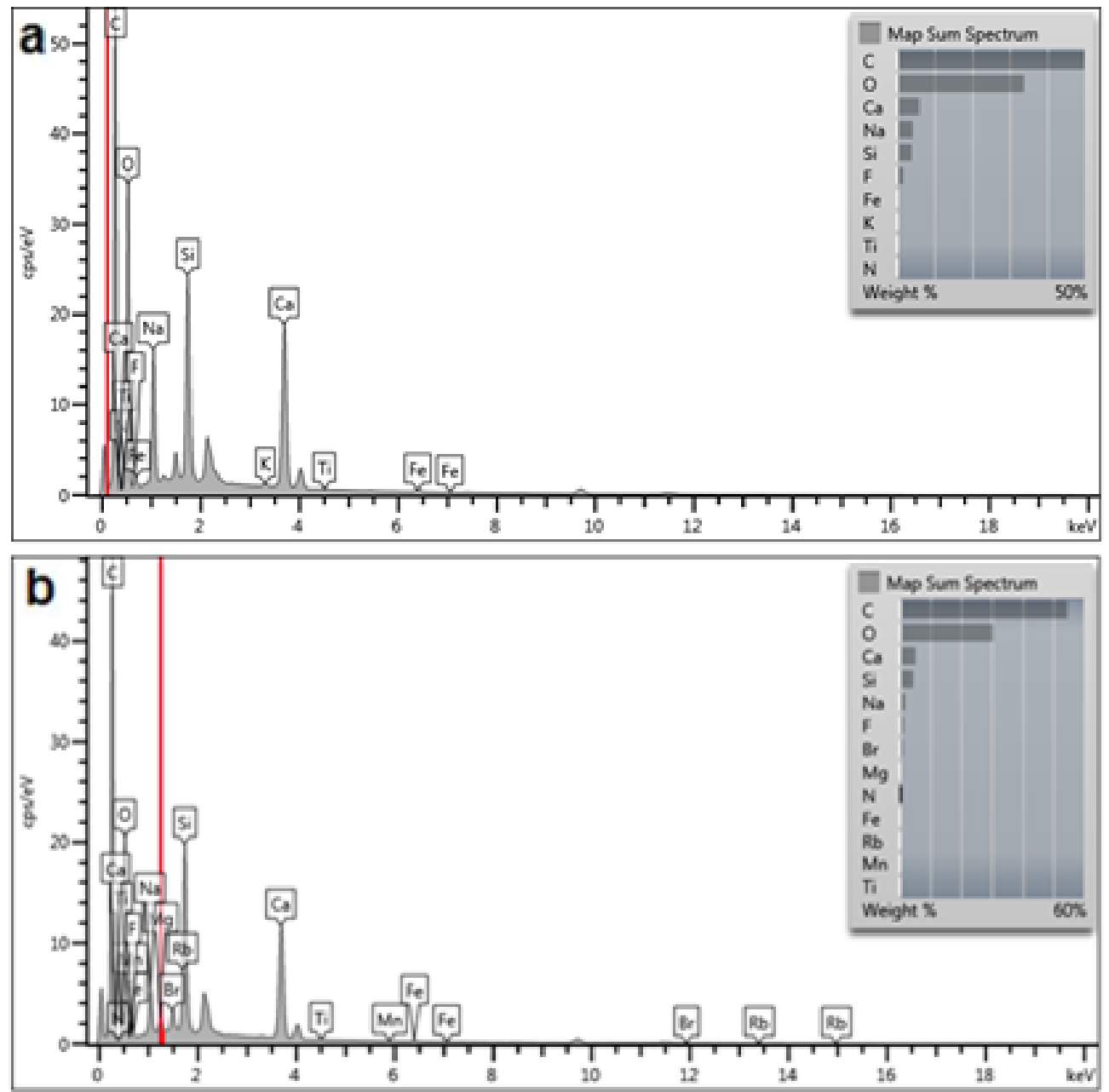

Figura 5. Espectro dos elementos químicos presentes nos pós fluxantes. (a) fluxante $A$ e (b) fluxante B.

Para obter-se uma precisa composição do fluxo o mais indicado é realizar análise por fluorescência de raios-X, contudo o EDS permite fazer uma pré-avaliação. Cruz et al. [18], explicam que na elaboração de um fluxante a adição de sílica e alumina na composição original favorece a formação de ligações aumentando a viscosidade e o ponto de fusão. Isto ocorre porque a sílica e a alumina são óxidos ácidos e agem como formadores de ligações quando o fluxo é fundido.

* Contribuição técnica ao 450 Seminário de Aciaria - Internacional, 25 a 28 de maio de 2014, Porto Alegre, RS, Brasil. 

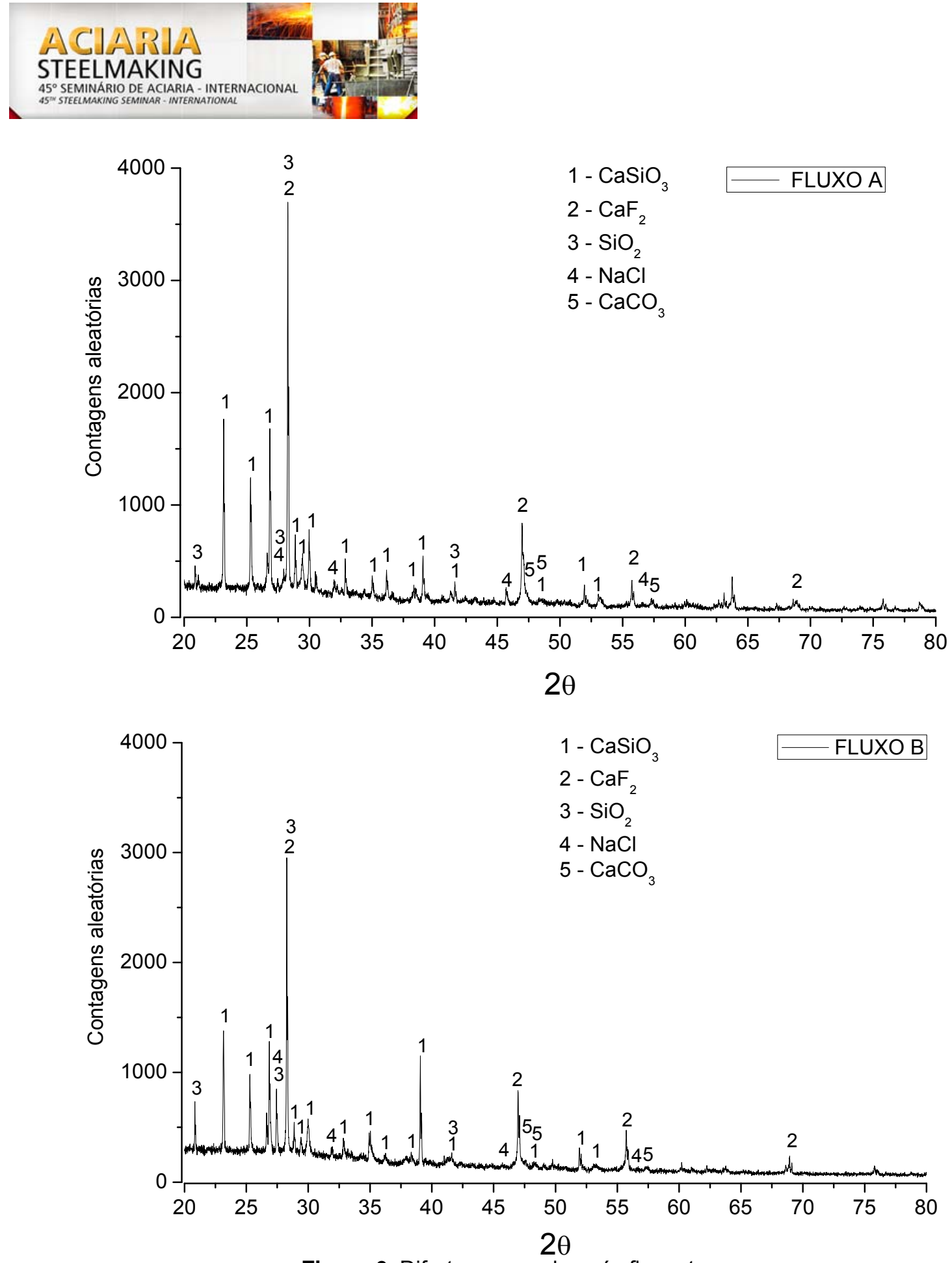

Figura 6. Difratogramas dos pós fluxantes.

Existem os constituintes que tendem a modificar as ligações entre as moléculas do fluxo como os fluoretos (ex.: $\mathrm{NaF}$ e $\mathrm{CaF}_{2}$ ), os alcalinos (ex.: $\mathrm{Na}_{2} \mathrm{O}, \mathrm{K}_{2} \mathrm{O}$ ) e os óxidos básicos (ex: $\mathrm{CaO}, \mathrm{MgO}, \mathrm{BaO}, \mathrm{SrO}$ ) todos reduzem a viscosidade através da diminuição do ponto de fusão total do sistema. O que se sabe é que sob aquecimento a tendência é que os constituintes originais que formam o fluxo reajam entre si e acabam por formar fases mais complexas tais como a já citada cuspidina $\left(3 \mathrm{CaO} .2 \mathrm{SiO}_{2} \cdot \mathrm{CaF}_{2}\right)$, a pectolita $\left(\mathrm{Na}_{2} \mathrm{CaSi}_{3} \mathrm{O}_{8}\right)$, e a pseudo-wolastonita $\left(\mathrm{CaSiO}_{3}\right)$. A presença de alumina irá favorecer a formação de anortita $\left(\mathrm{CaAl}_{2} \mathrm{Si}_{2} \mathrm{O}_{8}\right)$, gelenita $\left(\mathrm{Ca}_{2} \mathrm{Al}_{2} \mathrm{SiO}_{7}\right)$ e nefelina $\left(\mathrm{NaAlSiO}_{4}\right)$. A formação de todas estas fases afetam diretamente as condições de transmissão de calor, a viscosidade do filme líquido entre a placa e o molde e por fim afeta a velocidade de lingotamento. Prever

\footnotetext{
* Contribuição técnica ao 450 Seminário de Aciaria - Internacional, 25 a 28 de maio de 2014, Porto Alegre, RS, Brasil.
} 
exatamente como tudo isto irá interagir com processo e maximizar a produção é uma tarefa complexa.

A Figura 6 apresenta os difratogramas que indicam a presença das fases originais esperadas. A presença do Na favorece a formação da Nefelina e o silicato de sódio $\left(\mathrm{Na}_{2} \mathrm{SiO}_{3}\right)$. São compostos favoráveis para o lingotamento de aços com baixo teor de carbono onde a temperatura ótima de solidificação deverá estar entre 1040 e $1060^{\circ} \mathrm{C}$ e tendo-se a formação da Nefelina e do silicato de sódio inibi-se a formação da cuspidina que tem um elevado ponto de fusão. Ainda para favorecer o abaixamento do ponto de fusão é comum fazer a adição da fluorita, no entanto quando esta é adicionada acima de $12 \% \circ \mathrm{CaF}_{2}$ age como formador de ligações e isto favorece novamente a formação da cuspidina [18], observações práticas no chão de fábrica mostram que este limite pode ser menor chegando a $9 \%$. Provavelmente este é o comportamento do Fluxante A sendo indicado para aços peritéticos.

As Figuras 7 e 8 apresentam as análises térmicas diferencias e termogravimétricas respectivamente. Os resultados mostram que tanto o fluxante $A$ quanto o fluxante $B$ possuem picos endo e exotérmicos entre $450^{\circ} \mathrm{C}$ e $900^{\circ} \mathrm{C}$ que podem estar associados a decomposição de carbonetos queima de carbono livre e sinterização dos particulados. A Figura $8 \mathrm{com}$ a curva TG reforça que a decomposição dos carbonetos inicia-se por volta dos $450^{\circ} \mathrm{C}$ para ambos os fluxantes e que existe início de perda de massa expressiva também por volta de $1100^{\circ} \mathrm{C}$ indicando outras reações ainda não bem esclarecidas. Para o primeiro aquecimento, o último pico endotérmico ocorre a aproximadamente a $1247^{\circ} \mathrm{C}$ para o fluxante $\mathrm{A}$ e $1091^{\circ} \mathrm{C}$ para o fluxante $B$ indicando que o fluxante $A$ demora mais tempo para que seus constituintes reajam entre si desfavorecendo a fluidez. Este resultado era esperado porque o fluxante $A$ possui maior basicidade e maior quantidade de fluorita e conforme já dito anteriormente no parágrafo anterior o excesso de $\mathrm{CaF}_{2}$ pode favorecer a formação da cuspidina que tem elevado ponto de fusão. Este resultado é diferente do previsto pela simulação obtida pelo software Thermocalc $®$ que indicou completa fusão a $1305^{\circ} \mathrm{C}$ para o fluxante $\mathrm{A}$ e $1313^{\circ} \mathrm{C}$ para o fluxante $\mathrm{B}$. Ainda na Figura 7 temos resultados de reaquecimento dos fluxos que foram feitos imediatamente após a primeira fusão sob as mesmas condições. As curvas mostram picos referentes a novas reações e o último pico endotérmico para última curva de aquecimento indicam temperatura de fusão da ordem de $1252^{\circ} \mathrm{C}$ para o fluxante $\mathrm{A} \mathrm{e}$ $1279^{\circ} \mathrm{C}$ para o fluxante B. A questão cinética talvez seja um fator que explique as diferenças entre o previsto pelo Thermocalc® e as medições usando o DSC. No caso do primeiro aquecimento, talvez o tempo sob elevada temperatura não tenha sido suficiente para que se estabeleça as condições de equilíbrio previstas pelo software. As refusões posteriores acabam por trazer resultados mais aproximados já que os fluxos são mantidos sob aquecimento maior número de vezes. A simulação termodinâmica mostra que a completa fusão do fluxante $B$ se dá a aproximadamente $1313^{\circ} \mathrm{C}$ ou seja, 8 graus acima do fluxante $A$ e os resultados usando o DSC mostram a mesma tendência, porém são $27^{\circ} \mathrm{C}$ de diferença. Watanabe et al. [5], mostrou em seu trabalho que os fluxantes que possuem tendência de formar cuspidina durante o aquecimento irão apresentar um forte pico endotérmico referente a temperatura eutética seguido de um segundo pico mais fraco indicando o ponto de fusão ou a temperatura liquidus. Conforme pode ser observado na Figura 7 esta característica é muito similar ao fluxante $A$. O segundo e terceiro aquecimento mostram temperatura eutética de $1183^{\circ} \mathrm{C}$ e $1204^{\circ} \mathrm{C}$ respectivamente logo, adequado para ligas peritéticas.

\footnotetext{
* Contribuição técnica ao 450 Seminário de Aciaria - Internacional, 25 a 28 de maio de 2014, Porto Alegre, RS, Brasil.
} 


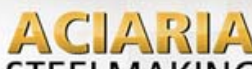
STEELMAKING

$45^{\circ}$ SEMINARIO DE ACIARIA-INTERNACIONAL
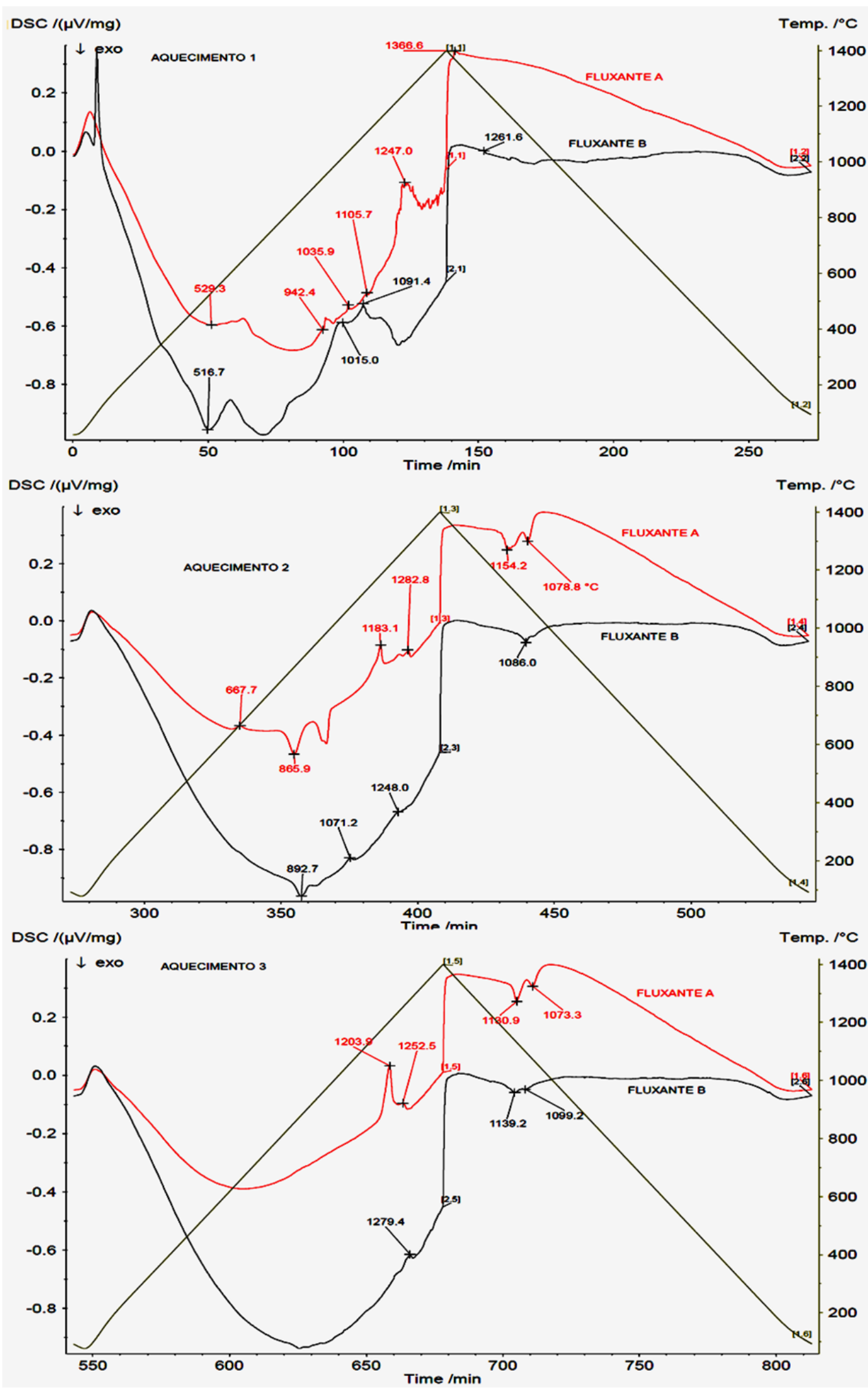

Figura 7. Análise térmica diferencial DSC dos fluxantes.

* Contribuição técnica ao 450 Seminário de Aciaria - Internacional, 25 a 28 de maio de 2014, Porto Alegre, RS, Brasil. 

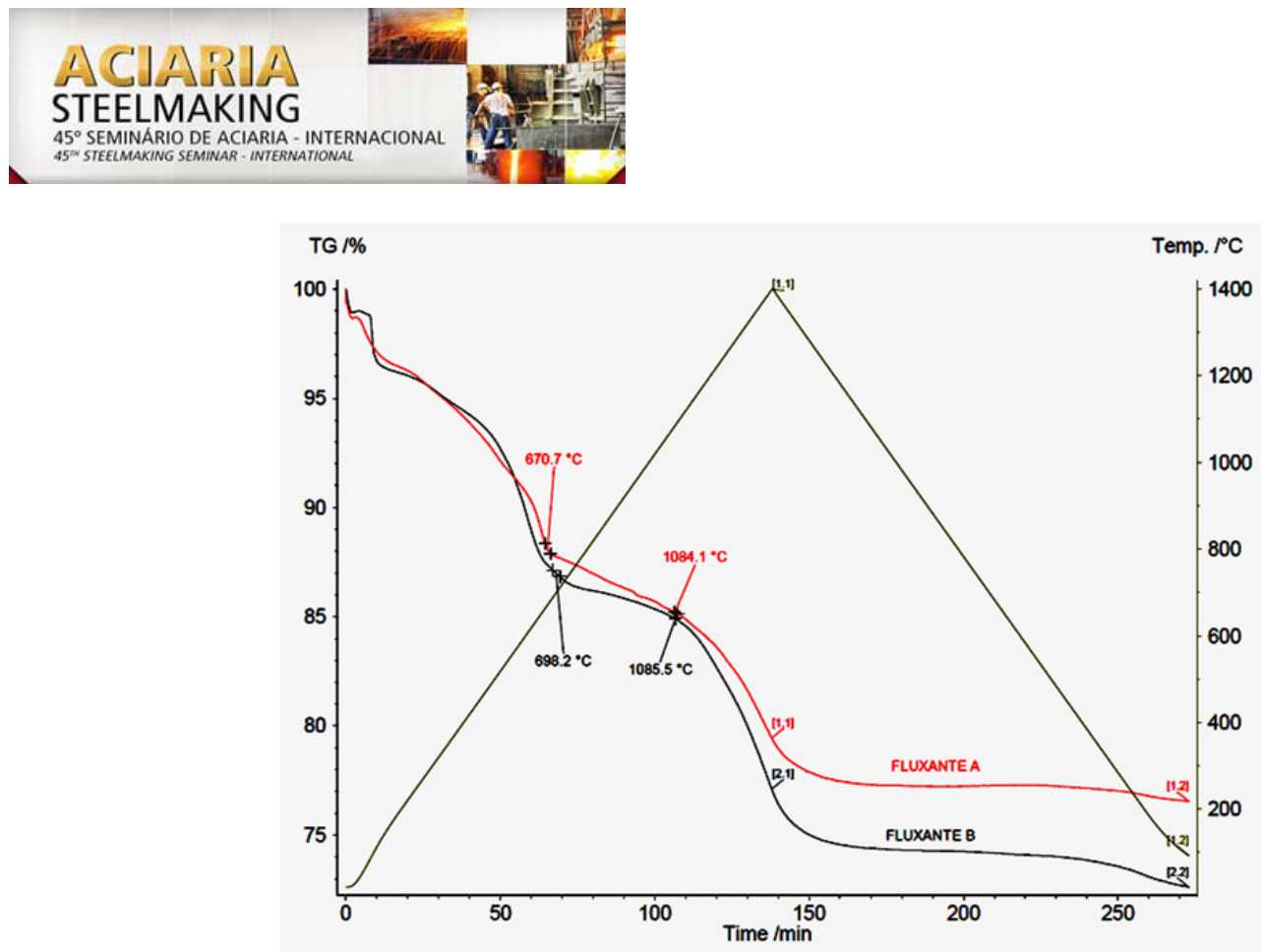

Figura 8. Análise térmica diferencial TG dos fluxantes.

\section{CONCLUSÃO}

O desenvolvimento de fluxantes para lingotamento contínuo visando à maximização da produtividade é uma tarefa complexa. Porém, a caracterização destes materiais por difração de raios-x, MEV/EDS e simulação computacional podem permitir prever como será o comportamento de novos materiais numa planta industrial. Para desenvolver novos fluxantes os dados aqui obtidos neste trabalho podem ser comparados com novas formulações. A caracterização e simulação computacional poderá permitir prever se estas novas composições irão atender os requisitos para uma determinada aplicação específica. Em trabalhos futuros os resultados experimentais de medidas de viscosidade, de difração de raios- $x$ para amostras submetidas ao aquecimento e resfriamento sob taxas variadas e análise térmica diferencial poderão ser confrontados com os dados obtidos por simulação computacional e pré-análise das matérias primas permitindo desenvolver novos fluxantes com características reológicas e térmicas previsíveis. Portanto, aqui foram apresentadas algumas ferramentas que podem ser usadas no processo de seleção de fluxantes e neste caso específico os resultados apontam para um tipo a ser usado no lingotamento de aços baixo carbono (fluxante B) e outro para aços peritéticos (fluxante A).

\section{Agradecimentos}

Os autores agradecem pelo apoio financeiro das agências de fomento Brasileiras: FAPES, CNPq e FINEP.

\section{REFERÊNCIAS}

1 Watanabe T, Fukuyama $\mathrm{H}$, Susa $\mathrm{M}$, Nagata $\mathrm{K}$. Phase diagram cuspidine $\left(3 \mathrm{CaO} .2 \mathrm{SiO}_{2} . \mathrm{CaF}_{2}\right)-\mathrm{CaF}_{2}$, Metallurgical and Materials Transactions B. 2000;31:12731281.

2 Meng Y, Thomas B.G.Modeling transient slag-layer phenomena in the shell/mold gap in continuous casting of steel, Metallurgical and Materials Transactions B. 2003;34B:707725.

* Contribuição técnica ao 45 Seminário de Aciaria - Internacional, 25 a 28 de maio de 2014, Porto Alegre, RS, Brasil. 
3 Görnerup M, Hayashi M, Däcker CA, Seetharaman S. Mould fluxe in continuous casting of steel - characterization and performance tuning. In: VII International Conference on Molten Slags Fluxes and Salts; 2004; Cape Town, South Africa. p.745-752.

4 Grill A, Brimacombe JK. Influence of carbon content on rate of heat extraction in the mould of a continuous-casting machine, Ironmaking and Steelmaking. 1976;3(2):76-79.

5 Fukuyama, $\mathrm{H}$. Tabata $\mathrm{H}$, Nagata K. Determination of gibbs energy of formation of cuspidine $\left(3 \mathrm{CaO} .2 \mathrm{SiO}_{2} . \mathrm{CaF}_{2}\right)$ from the electromotive force method using $\mathrm{CaF}_{2}$ as the solid electrolyte. Metallurgical and Materials Transactions B. 2003;34:307-311.

6 Cho JW, Shibata H, Emi, T, Suzuki M. Thermal resistance at the interface between mold flux film and mold for continuous casting of steels. Iron and Steel Institute of Japan. 1998;38(5):440-446.

7 Cho JW, Shibata H, Emi T, Suzuki M. Heat transfer across mold flux film in mold during initial solidification in continuous casting of steel. Iron and Steel Institute of Japan. 1998;38(8):834-842.

8 Watanabe K, Suzuki M, Murauchi K, Kondo H, Miyamoto A, Shiomi T. The Effect of crystallization of mold powder on the heat transfer in continuous casting mold, Tetsu-toHagané. 1997;83(2):115-120.

9 Kashiwaya Y, Cicutti CE, Cramb AW. An investigation of the crystallization of a continuous casting mold slag using the single hot thermocouple technique. Iron and Steel Institute of Japan. 1998;38(4):357.

10 Tsutsumi K, Ohtake J, Nagasaka T, Hino M. Crystallization Behavior of $\mathrm{Li}_{2} \mathrm{O}-\mathrm{SiO}_{2}, \mathrm{Na}_{2} \mathrm{O}-$ $\mathrm{SiO}_{2}$ and $\mathrm{Na}_{2} \mathrm{O}-\mathrm{CaO}-\mathrm{SiO}_{2}$ Glasses. Tetsu-to-Hagané. 1998;84(6):464-469.

11 Lanyi MD, Rosa CJ. Viscosity of casting fluxes used during continuous casting of steel. Metallurgical Transactions B. 1981,12B:287-298.

12 Meng $Y$, Thomas BG. Modeling transient slag-layer phenomena in the shell/mold gap in continuous casting of steel. Metallurgical Transactions B. 2003;34B:707-725.

13 Thresh HR, Crawley AF. Viscosities of lead, tin and Pb-Sn alloys. Metallurgical Transactions. 1970;1:1531.

14 Ganesan S, Speiser R, Poirier DR. Viscosities of aluminum-rich Al-Cu liquid alloys. Metallurgical Transactions B. 1987;18B:421-725.

15 Seetharaman S, Sichen D. Viscosities of High temperature systems - a moelling approach. Iron and Steel Institute of Japan. 1997;37(2):109-118.

16 Moelwyn-Hughes EA. Physical Chemistry. Oxford: Pergamon Press; 1961.

17 Dey A, Riaz S. Viscosity measurement of mould fluxes using inclined plane test and deveopment of mathematical model. Ironmaking and Steelmaking. 2012;39(6):391-397.

18 Cruz A, Chávez F, Romero A, Palacios E, Arredondo V. Mineralogical phases formed by flux glasses in continuous casting mould. Journal of Materials Processing Technology. 2007; 182:358-362.

* Contribuição técnica ao $45^{\circ}$ Seminário de Aciaria - Internacional, 25 a 28 de maio de 2014, Porto Alegre, RS, Brasil. 